\title{
Serum Level of Fibroblast Growth Factor 23 in Patients with Uremic Pruritus Undergoing Hemodialysis
}

\author{
A.I.El-Taweel ${ }^{1,}$ A.I.Mustafa ${ }^{1}$, A.E.Mansour ${ }^{2}$, R.A.Khashba ${ }^{3}$ and N.K.Abd elSalam ${ }^{1}$ \\ ${ }^{1}$ Dermatology, Venereology and Andrology Dept., Faculty of Medicine, Benha Univ, Benha, Egypt \\ ${ }^{2}$ Clinical and Chemical Pathology Dept., Faculty of Medicine, Benha Univ, Egypt \\ ${ }^{3}$ Internal Medicine Dept, Faculty of Medicine, Benha Univ, Egypt \\ E-mail: Lily_movie_star@hotmail.com
}

\begin{abstract}
Uremic pruritus is a common and intractable symptom in patients on chronic hemodialysis. It causes serious discomfort and skin damage, negatively affects the quality of life, and may be associated with sleep disturbance, inflammation, and higher mortality.The aim of the present study was to assess serum level of Fibroblast growth factor 23 [FGF 23] in patients with uremic pruritus undergoing hemodialysis to evaluate its role in uremic pruritus pathogenesis and evaluation of its clinical significance. The present study was conducted as a comparative cross-sectional study, study included 80 patients with end stage renal disease undergoing hemodialysis; 40 of them were suffering from uremic pruritus [Group A] and 40 patients were not suffering from it [GroupB]. All patients were recruited from the hemodialysis unit of Benha University Hospital between May 2017 and July 2018, All studied subjects were tested for serum level of endothelin-1 [ET-1] 1 in both patients groups using an ELISA technique.There was no significant difference of the serum level of FGF 23 regarding age, biochemical parameters and VAS score of pruritus. While, the duration of hemodialysis was significantly longer among group A patients [p 0.001].Fibroblast growth factor 23 may play a role in the pathogenesis of uremic pruritus and can be used as a sensitive biomarker in predicting uremic pruritus in chronic kidney disease patients undergoing hemodialysis.
\end{abstract}

Keywords: Hemodialysis, Pruritus, Uremic, FGF 23.

\section{Introduction}

Uremic pruritus is a as a relatable point Furthermore immovable manifestation On patients ahead incessant hemodialysis. It makes genuine uneasiness What's more skin damage, negatively influences the nature from claiming life, What's more might be connected with rest disturbance, inflammation, and higher mortal sin [1].

Those pathophysiology about uremic pruritus is unpredictable. Past investigations have indicated that xerosis, divalent ions, calcium-phosphate product, Creactive protein, hepatitis, hyperparathyroidism, resistant derangement, What's more opioid framework rotation might a chance to be connected with uremic pruritus [2].

Due to insufflate Comprehension for uremic pruritus, present restorative choices to uremic pruritus are restricted and unsuitable. Huge numbers patients have a prolonged course for uremic pruritus. Inparticular, 30-60\% from claiming patients looking into upkeep hemodialysis sufferfrom this issue to longer over you quit offering on that one quite a while [3].

Fibroblast development factor-23 [FGF23] will be a circle part of the FGF gang handled primarily Eventually Tom's perusing the osteocytes and osteoblasts that might go about as a hormone. Those principle movement of FGF23 will be with bring down phosphatemia through the diminishment of urinary phosphate reabsorption and the decline for $1,25[\mathrm{OH}] 2$ $\mathrm{D}$ era in the kidney. In the span from claiming Ceaseless kidney sickness [CKD], plasma FGF23 centralization ascents early, The majority likely will adjust those powerlessness of the deteriorating kidneys will discharge an sufficient sum about phosphate. However, this goes at the expense about FGF23-related target organ poisonous quality. [1]. Comes about for clinical investigations propose that raised plasma FGF23 fixation is freely connected with the expanded hazard for CKD progression, event of cardio-vascular complications, and mortal sin in distinctive phases about CKD. "around the variables expanding plasma FGF23 concentration, animated vitamin $d$ analogues assume a significant part. Additionally, inflammation Also iron deficiency camwood help those build of plasma FGF23. "around those elements diminishing plasma FGF23, dietary phosphate restriction, A percentage intestinal phosphate binders, cinacalcet [and other calcimimetics], Also nicotinamide could be enumerated.

There need been a continuous exchange something like if constant tingle over incessant kidney sickness may be achieved Eventually Tom's perusing those regular aggravation for calcium/phosphate homeostasis. In the late 1960s, tingle might have been showed up for be determined by parathyreoidectomy over patients for both extreme hyperparathyroidism What's more incessant tingle. [4,5]. accounted an expanded calcium/phosphate load in the skin of patients with constant tingle to hemodialysis What's more were unable should exhibit a disturbed calcium gradient in the skin for patients with Ceaseless tingle. An observational investigation discovered that patients for incessant tingle required higher serum-calcium levels. It need been suggested as of late that An horrendous circis siliquastrum of metabolic derangements [malnourishment, inflammation, arteriosclerosis] might demonstrate the misrepresented horribleness What's more mortal sin over a subset of hemodialysis patients. Aggravation may be the A large portion injurious calculate in this scenario, contributing, Around other factors, of the event for Ceaseless tingle for hemodialysis.

Large amounts from claiming fibroblast growthfactor 23 [FGF23], An intense player over 
calcium/phosphate-regulation, appear to be with make connected with expanded aggravation Furthermore horribleness and mortal sin to patients with Ceaseless kidney sickness [CDK] [6].

\section{Patients and methods}

2.1Type of study

The present study was conducted as a comparative cross-sectional study.

\section{Study Population}

This study included 80 patients with end stage renal disease undergoing hemodialysis; 40 of them were suffering from uremic pruritus [Group A] and 40 patients were not suffering from it [GroupB]. All patients were recruited from the hemodialysis unit of Benha University Hospital between May 2017 and July 2018.

\subsection{Ethical Considerations}

The study was approved by the local ethics committee on research involving human subjects of Benha Faculty of Medicine. Informed consents were taken from all participants before taking any data or doing any laboratory investigations.

\subsection{Inclusion criteria}

Patients on regular HD three times weekly and suffering from uremic pruritus.

\subsection{Exclusion criteria}

Any patient was excluded if he/she had:

Systemic pruritic diseases such as hepatitis C, B virus infection and allergic diseases.
2.5 Methods: Each patient will be subjected to the following:

\section{A. History of renal disease:}

Onset, course and duration of renal failure, history of underlying diseases [Hypertension, DM or SLE], medications and duration of HD.

\section{Laboratory Investigations}

(A) Assessment of serum Glutamic Oxalo Acetic Transaminase [SGOT], serum Glutamic Pyruvate Transaminase [SGPT], urea, creatinine, uric acid [UA], calcium[Ca], phosphorus $[\mathrm{P}]$, parathyroid hormone [PTH] and hemoglobin levels $[\mathrm{Hb}]$ in both patients groups.

(B) Estimation of serum level of Endothelin-1:

- All studied subjects were tested for serum level of endothelin-1 [ET-1] 1 in both patients groups using an ELISA technique.

- Sampling: Three ml venous blood was collected from each subject under complete aseptic technique after 6-8 hours fasting by clean venipuncture using disposable plastic syringe and placed on plain tube [without anticoagulant] for serum separation. The tube was left at room temperature for 30 minutes till coagulation, and then was centrifuged [at $1500 \mathrm{rpm}$ for 15 minutes]. The resultant serum was divided ino 3 aliquots and stored at $\mathbf{- 2 0}{ }^{\circ} \mathrm{C}$ for further testing.

\subsection{Statistical analysis of the data}

Data were fed to the computer using IBM SPSS software package version 20.0.

Qualitative data were described using number and percent. Comparison between different groups regarding categorical variables was tested using Chi-square test.

\section{Results}

Table (1) Comparison between the two studied groups regarding FGF.

\begin{tabular}{lccc}
\hline & $\begin{array}{c}\text { Group I } \\
\text { "patients" }\end{array}$ & $\begin{array}{c}\text { Group II } \\
\text { "control" }\end{array}$ & P \\
\hline FGF & $119.2-940.1$ & $52.1-209.1$ & $0.001 *$ \\
Range & $417.22 \pm 210.84$ & $133.74 \pm 45.51$ & \\
\hline
\end{tabular}

The mean serum level of FGF 23 was significantly higher in group [A] than group [B] patients [p<0.001] as shown in Table (1).

\section{Discussion}

Uremic pruritus will be a normal and immovable side effect for patients once unending hemodialysis. It reasons not kidding uneasiness What's more skin damage, negatively influences the personal satisfaction from claiming life, and might be connected with rest disturbance, inflammation, Also higher mortal sin [1].

Those pathophysiology from claiming uremic pruritus may be perplexing. Past investigations have indicated that xerosis, divalent ions, calcium-phosphate product, C-reactive protein, hepatitis, hyperparathyroidism, safe derangement, and opioid framework rotation might make connected with uremic pruritus [2].

Fibroblast development factor-23 [FGF23] may be a circle part of the FGF crew transformed primarily Toward those osteocytes Furthermore osteoblasts that camwood go about as An hormone. The principle movement about FGF23 may be to easier phosphatemia through those decrease for urinary phosphate reabsorption and the diminishing from claiming $1,25[\mathrm{OH}] 2-\mathrm{D}$ era in the kidney. In the course of incessant kidney sickness [CKD], plasma FGF23 fixation climbs early, The greater part Presumably to 
adjust those powerlessness of the deteriorating kidneys on discharge a sufficient measure about phosphate. However, this goes toward the expense for FGF23related target organ poisonous quality. [1]. There need been a progressing dialog over if constant tingle Previously, incessant kidney sickness will be achieved Toward the as a relatable point Unsettling influence from claiming calcium/phosphate homeostasis. In the late 1960s, tingle might have been appeared for be determined Toward parathyreoidectomy clinched alongside patients for both extreme hyperparathyroidism Also unending tingle.

The point for this investigation might have been on survey serum level for fibroblast development element 23 to patients for uremic pruritus undergoing hemodialysis Furthermore assessment of its clinical noteworthiness.

This consider included 80 patients for conclusion stage renal malady undergoing hemodialysis; 40 of them enduring starting with uremic pruritus [Group An] Also 40 patients not suffice from uremic pruritus [Group B]. Every last bit patients were chosen from the hemodialysis unit of Benha school healing facilities. Composed educated agrees were acquired starting with all members. Those ponder might have been endorsed Eventually Tom's perusing those morals panel once examination directing, including human subjects of Benha employees of solution.

In this study, the demographic information of the two contemplated bunches demonstrate inconsequential distinction in regards agdistis and sex, this comes about might have been paramount with kill those impact from claiming demographic information on the net outcomes.

Clinched alongside our study the blood picture [WBCs, hb Also platelet] in the two gatherings indicate inconsequential difference, this comes about might have been matched with consider conveyed crazy Eventually Tom's perusing Ozen et al. , 2018, in this ponder it might have been discovered that there might have been no huge distinction between those uremic pruritus Furthermore non uremic.

As opposed will our effects Kimata et al discovered that higher WBC checks expanded the danger about dependent upon advancement by 1. 04-fold [7]. Similarly, Pisoni et al. Found that a WBC check > 8. $4 \times$ $103 / \mu \mathrm{L}$ expanded the hazard for up create ment Eventually Tom's perusing 1. 20-fold [8].

Previously, our ponder the A long time from claiming HD for bunch i went from 2 - 25 with mean quality $9.75 \pm 4$. 63 Furthermore to gathering ii went starting with $1-16$ for mean quality $6.18 \pm 3.71$. There might have been Factual noteworthy distinction the middle of the two concentrated on gatherings in regards HD $[\mathrm{P}<0.05]$. This comes about might have been concurrence with An multi-center contemplate conveyed out Eventually Tom's perusing Ozen et al. , 2018, in this ponder he discovered that the in length span for dialysis related for uremic pruritus. [9].

\section{Conclusion}

Fibroblast Growth figure 23 might assume a part in the pathogenesis from claiming uremic pruritus and could make utilized Concerning illustration An touchy biomarker Previously, foreseeing uremic pruritus in Ceaseless kidney sickness patients undergoing hemodialysis.

\section{References}

[1] M.I.Duque, S.Thevarajah, Y.H.Chan: Uremic pruritus is associated with higher $\mathrm{kt} / \mathrm{V}$ and serum calcium concentration. Clin. Nephrol, Vol. 66,PP. 184-191,2006.

[2] Y.Chen, W. Chiu, Therapeutic effect of topical gamma-linolenic acid on refractory uremic pruritus. The American Journal of Kidney Diseases, Vol. 48, PP. 69-76,2006.

[3] I.Zucker, G.Yosipovitch, M.David, Prevalence and characterization of uremic pruritus in patients undergoing hemodialysis: uremic pruritus is still a major problem for patients with end-stage renal disease. Journal of the American Academy of Dermatology, Vol. 49(5), PP. 842-846,2003.

[4] G.Virga, I.Visentin, V.L.Milia, Inflammation and pruritus in haemodialysis patients. Nephrol Dial Transplant vol .17,PP.2164-2169,2002

[5] A.Momose, S.Kudo, M.Sat, Calcium ions are abnormally distributed in the skin of haemodialysis patients with uraemic pruritus. Nephrol Dial Transplant, Vol.19 PP.2061-2066.2004.

[6] P.Kuczera, M.Adamczak, A.Wiecek, Fibroblast Growth Factor-23-A Potential Uremic Toxin, Department of Nephrology, Transplantation and Internal Medicine, Medical University of Silesia,Vol.8, PP.2171.2016.

[7] N.Kimata, D.S.Fuller, A.Saito,, Pruritus in hemodialysis patients: Results from the Japanese Dialysis Outcomes and Practice Patterns Study [JDOPPS]. Hemodial Int vol18, PP.657- 667, 2014.

[8] R.Pisoni, B.Wikstrom, S.Elder, Pruritus in haemodialysis patients: international results from the Dialysis Outcomes and Practice Patterns Study [DOPPS]. Nephrology Dialysis Transplantation, Vol. 21(12), PP. 3495-3505.2006.

[9] N.Ozen, F.Cinar, D.Askin, Uremic pruritus and associated factors in hemodialysis patients: A multi-center study. Kidney Res Clin Pract Vol.37 ,PP.138-147.2018. 